Research Article

\title{
Event-Triggered Controller Design for Autopilot with Input Saturation
}

\author{
Guoqing Xia, Xiaoming Xia $(\mathbb{D}$, Zhao Bo, Xianxin Sun $(\mathbb{D}$, and Chuang Sun \\ College of Automation, Harbin Engineering University, Harbin 150001, China \\ Correspondence should be addressed to Xiaoming Xia; xia_xm@126.com
}

Received 14 December 2019; Revised 7 April 2020; Accepted 30 May 2020; Published 16 June 2020

Academic Editor: Zhan Shu

Copyright (C) 2020 Guoqing Xia et al. This is an open access article distributed under the Creative Commons Attribution License, which permits unrestricted use, distribution, and reproduction in any medium, provided the original work is properly cited.

This paper considers the problem of autopilot design for surface ship with environmental disturbances and input saturation. Extended state observer (ESO) is used to reconstruct yaw rate and estimate unknown environmental disturbances. An auxiliary dynamic system is employed to handle the input saturation. By combining with the auxiliary dynamic system and ESO, an eventtriggered controller (ETC) is designed, and energy is saved by reduction in the rudder rate. Simulation results illustrate the effectiveness and feasibility of the event-triggered proposed controller.

\section{Introduction}

Autopilot design of marine surface vehicles has witnessed a surge of interest. Autopilot design with sophisticated controllers has a wide application prospect in reduced manpower, adequate economy, sufficient reliability, and optimum performance [1].

A significant amount of research efforts has been focused on autopilot. In the beginning, because of simple structure and preferable antinoise ability, proportional integral differential (PID) algorithm is applied to design autopilot. However, the parameters for the PID control terms are not easy to tune. To solve these problems [2], consider a class of optimal PID control problems, each of these optimal parameter selection problems is solved as a nonlinear optimization problem [3], and present an adaptive PID control algorithm which improves the grey predictive control for temperature control. In [4], a nonlinear function is employed to describe manoeuvring characteristics that reflect the steady-state relation between the rudder deflection and the rate of turn of the hull, and two configurations of nonlinear controllers are applied to ship course.

In practice, the actual yaw angle is measured from the sensors or observer [5]. Das [6] makes an assumption that the yaw and yaw rate are all measurable, which is difficult to implement. Peng et al. [7] present a robust adaptive steering law based on dynamic surface control technique and neural network, and a state observer is employed to reconstruct the yaw rate.

Environmental disturbances and model uncertainties are important challenges. Rigatos and Tzafestas [8] propose an adaptive fuzzy $H_{\infty}$ controller for the autopilot system, and unknown part of the system dynamics can be approximated with the indirect adaptive control. Liu et al. [9] propose an output feedback adaptive steering law for an unmanned surface vehicle, and a neural network using iterative updating law is employed to handle the unknown dynamic and environmental disturbances. A sliding mode control strategy for the autopilot system is proposed in [10], where a fuzzy logic is used to handle gain and avoid the chattering effect. Xia et al. [11] address the problem of adaptive neural network controller for the surface vessel, and adaptive technique and neural network system are used to approximate uncertainties. Disturbance observer is employed to estimate the unmodeled external forces in $[12,13]$.

All of the above articles do not consider the limitation of rudder angle, which is widespread in practice. Input saturation degrades the quality of the control and can even destabilize the system. Hence, it is necessary to consider the limitation of the rudder angle. Tzeng and Lin [14] propose an adaptive ship steering approach which is characterized by a model-based design approach that provides clear 
connections between the structure of the controller and ship model. Babaei et al. [15] present an efficient strategy to design the altitude hold mode autopilot for unmanned aerial vehicle, and a multiobjective genetic algorithm is used to mechanize the optimal determination of fuzzy logic controller parameters.

In many applications, rudder limits includes magnitude limits and rate limits. The digital computer with sampler and zero-order holder is employed to emulate the continuoustime control scheme, and the control command will be updated periodically [16]. Katayama and Aoki [17] propose the use of a performance-based supervisory switching control system for trajectory tracking unmanned surface vessels without considering the actuator magnitude limits and rate limits. The performance-based simulator includes the effects of actuator rate limits and saturation, and the results show that the orientation cannot stabilize to the tangent velocity. Furthermore, high-frequency action will lead to the mechanical abrasion and increase fuel consumption. Shipping is no different than other industries and is highly affected by fuel prices [18]. Thus, it is practical to lower the acting frequencies of actuators. An event-triggered robust fuzzy control scheme is developed for underactuated ships in [19], where fuzzy logic systems are employed to approximate the uncertainties, and the ideology of ETC is employed to avoid the frequent acting of actuators.

This paper reports an autopilot design for surface vehicle, subject to environmental disturbances and input saturation. Extended state observer (ESO) is employed to estimate the unknown environmental disturbances. An auxiliary dynamic system is designed to handle the input saturation. The control architecture is developed by ESO, auxiliary dynamic system, and event-triggered strategy. By using the event-triggered strategy, the proposed approach is able to reduce the rudder rate, which has great significance to the reduction of mechanical wear and fuel consumption. The stability analysis using Lyapunov function shows that all error signals in the closed-loop system are uniformly ultimately bounded. Simulation result shows the effectiveness of the proposed method.

This paper is organized as follows. Section 2 formulates the surface ship model with input saturation and the problem formulation. Section 3 depicts the design of the extended state observer. Section 4 presents the design of the controller by using the event-trigger strategy and auxiliary dynamic system, gives the stability analysis, and demonstrates the effectiveness of the proposed control scheme. Section 5 provides a simulation to illustrate the theoretical result. Section 6 concludes this paper.

\section{Mathematical Model}

2.1. Notation. The following notations will be used throughout this paper. |.| denotes the absolute value unless specified. $\|$.$\| represents the Euclidean norm. \mathbb{R}^{m \times n}$ represents the $m \times n$-dimensional Euclidean Space. $\lambda_{\text {min }}$ represents minimum eigenvalue of a square matrix. $(.)^{T}$ denotes the transpose of a matrix.
2.2. Model of the Ship. As shown in Figure 1, assume that the ship has an $x z$-plane of symmetry, and heave, pitch, and roll motions are neglected. The body-fixed frame coordinate origin is set in the center-line of the ship. The mathematical model of the ship moving in a horizontal plane is described as [20]

$$
\begin{aligned}
\dot{\eta} & =J(\psi) \nu, \\
M \dot{v} & =-C(\nu) \nu-D(\nu) \nu+d+\tau,
\end{aligned}
$$

where $\eta=[x, y, \psi]^{T}$ is the vector denoting the ship position $(x, y)$ and yaw angle $\psi$ with coordinates in the earth-fixed frame, $v=[u, v, r]^{T}$ is the vector denoting surge, sway, and yaw velocities of the ship in the body-fixed frame, $d=\left[d_{1}, d_{2}, d_{3}\right]^{T}$ is the vector representing environmental disturbances, and $\tau=\left[\tau_{u}, \tau_{v}, \tau_{r}\right]^{T}$ is the control vector of the ship which consists of the surge force $\tau_{u}$, sway force $\tau_{v}$, and yaw moment $\tau_{r}$. The matrices $J(\psi)$ is given by

$$
\begin{aligned}
J(\psi) & =\left[\begin{array}{ccc}
\cos (\psi) & -\sin (\psi) & 0 \\
\sin (\psi) & \cos (\psi) & 0 \\
0 & 0 & 1
\end{array}\right], \\
M & =\left[\begin{array}{ccc}
m-X_{\dot{u}} & 0 & 0 \\
0 & m-X_{\dot{v}} & m x_{g}-Y_{\dot{r}} \\
0 & m x_{g}-N_{\dot{v}} & I_{z}-N_{\dot{r}}
\end{array}\right],
\end{aligned}
$$

where $m$ is the mass of the ship, $I_{z}$ is the moment of inertia about $z$-axis in the b-frame, $x_{g}$ is the center of gravity in the body-fixed frame, and $X_{\dot{v}}, Y_{\dot{r}}, N_{\dot{v}}$, and $N_{\dot{r}}$ are the hydrodynamic parameters:

$$
C=\left[\begin{array}{ccc}
0 & 0 & c_{13} \\
0 & 0 & c_{23} \\
c_{31} & c_{32} & 0
\end{array}\right],
$$

where $c_{13}=-\left(m-X_{\dot{v}}\right) v-\left(m x_{g}-Y_{\dot{r}}\right) r, \quad c_{23}=\left(m-X_{\dot{u}}\right) u$, $c_{31}=\left(m-X_{\dot{v}}\right) v+\left(m x_{g}-Y_{\dot{r}}\right) r$, and $c_{32}=-\left(m-X_{\dot{u}}\right) u$. $C(\nu)=-C^{T}(\nu) \in \mathbb{R}^{3 \times 3}$ represents a skew-symmetric matrix of Coriolis and centripetal term:

$$
D=\left[\begin{array}{ccc}
d_{11} & 0 & 0 \\
0 & d_{22} & d_{23} \\
0 & d_{32} & d_{33}
\end{array}\right],
$$

where $\quad d_{11}=-\left(X_{u}+X_{u|u|}|u|\right), \quad d_{22}=-\left(Y_{v}+Y_{v|v|}|v|\right.$ $\left.+Y_{r|v|}|r|\right), \quad d_{23}=-\left(Y_{r}+Y_{|v| r \mid}|v|+Y_{|r| r}|r|\right), \quad d_{32}=-\left(N_{v}+\right.$ $\left.N_{|v| v}|v|+N_{|r| v}|r|\right), d_{33}=-\left(N_{r}+N_{|v| r}|v|+N_{|r| r}|r|\right)$, and $X_{u}$, $X_{u|u|}, Y_{v}, Y_{v|v|}, Y_{r|v|}, Y_{r}, Y_{|v| r}, Y_{|r| r}, N_{v}, N_{|v| v}, N_{|r| v}, N_{r}, N_{|v| r}$, and $N_{|r| r}$ are linear and quadratic drag coefficients.

2.3. Model Decoupling. The mathematical model represented by equation (1) can be simplified by supposing a constant surge speed $u=u_{0}$, The sway-yaw dynamics can be separated from model and is described as

$$
M_{v r} \dot{v}_{v r}+N\left(u_{0}\right) v_{v r}=\tau_{v r}
$$




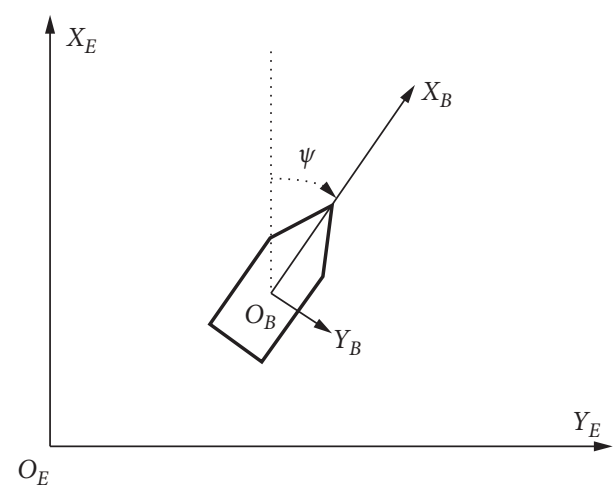

Figure 1: Body-fixed frame $X_{B} O_{B} Y_{B}$ and earth-fixed frame $X_{E} O_{E} Y_{E}$.

where $v_{v r}=[v, r]^{T}$ is the state vector, $\tau_{v r}$ is a vector which represents the force and moment in sway and yaw directions, respectively, and

$$
\begin{aligned}
M_{v r} & =\left[\begin{array}{cc}
m-X_{\dot{v}} & m x_{g}-Y_{\dot{r}} \\
m x_{g}-N_{\dot{v}} & I_{z}-N_{\dot{r}}
\end{array}\right], \\
N\left(u_{0}\right) & =\left[\begin{array}{cc}
-Y_{v} & -Y_{r}+\left(m-X_{\dot{u}}\right) u_{0} \\
-N_{v}+\left(X_{\dot{u}}-Y_{\dot{v}}\right) u_{0} & -N_{r}+\left(m x_{g}-Y_{\dot{r}}\right) u_{0}
\end{array}\right] .
\end{aligned}
$$

One notes that $N\left(u_{0}\right)$ can be attained by

$$
N\left(u_{0}\right)=C_{v r}+D_{L}
$$

where

$$
\begin{aligned}
C_{v r} & =\left[\begin{array}{cc}
0 & \left(m-X_{\dot{u}}\right) u_{0} \\
\left(X_{\dot{u}}-Y_{\dot{v}}\right) u_{0} & \left(m x_{g}-Y_{\dot{r}}\right) u_{0}
\end{array}\right], \\
D_{L} & =\left[\begin{array}{cc}
-Y_{v} & -Y_{r} \\
-N_{r} & -N_{r}
\end{array}\right] .
\end{aligned}
$$

Assume that the ship has a single rudder, and the following is obtained:

$$
\tau_{v r}=b \delta,
$$

where $b=\left[-Y_{\delta},-N_{\delta}\right]^{T}, Y_{\delta}$ and $N_{\delta}$ are the coefficients of force in sway and moment in yaw, respectively.

In this model, equation (5) can be transformed into Nomoto's model by eliminating the sway velocity written as

$$
\frac{r(s)}{\delta(s)}=\frac{K\left(1+T_{3} s\right)}{\left(1+T_{1} s\right)\left(1+T_{2} s\right)},
$$

where $T_{i}(i=1,2,3)$ is the parameter related to the characteristics of the ship, $r$ is the yaw rate, $\delta$ is the rudder angle, and $K$ is the gain constant related to the hydrodynamic coefficients. Experimentally, the constants $T_{2}$ and $T_{3}$ are nearly equal. Hence, equation (10) can be written as

$$
\frac{r(s)}{\delta(s)}=\frac{K}{1+T s},
$$

where $T=T_{1}+T_{2}-T_{3}$.
Consider input saturation, and it can be attained that $-U_{m} \leq \delta \leq U_{m}$, where $U_{m}$ is the maximum rudder deflection. Define the mismatch function between input without saturation and with saturation as $\sigma=\delta_{c}-\delta$, where $\delta_{c}$ is the rudder angle calculated by the controller.

2.4. Control Objective. The desired angle $\psi_{d}$ provided by the guidance system, in here, it is given directly. The desired yaw rate $r_{d}$ is the signals derived from the following second-order filter:

$$
\begin{aligned}
& \dot{\psi}_{a}=r_{d}, \\
& \dot{r}_{d}=\iota_{1}^{2}\left(\psi_{a}-\psi_{d}\right)-2 \iota_{1} r_{d},
\end{aligned}
$$

where $\iota_{1} \in \mathbb{R}$ is a positive constant.

The control objective is to force the surface vehicle to track a reference heading signal $\psi_{d}$ and lower the acting frequencies of actuators, such that

$$
\lim _{t \rightarrow+\infty}\left|\psi-\psi_{d}\right| \leq \varepsilon
$$

where $\varepsilon$ is a positive constant which can be made small enough.

\section{Extended State Observer}

The proposed autopilot controller is designed based on ESO which is designed to reconstruct the unmeasurable yaw rate, environmental disturbances, and model uncertainties.

Reshape dynamic (11) with the mismatch function to facilitate the controller design:

$$
\dot{r}=\frac{K}{T}\left(\delta_{c}-\sigma+\delta_{w}\right)-\frac{1}{T} r,
$$

where $\delta_{w}$ is unknown and bounded environmental disturbances.

In order to facilitate the ESO design, equation (14) is rewritten in the state space form as

$$
\begin{aligned}
& \dot{x}_{1}=x_{2}, \\
& \dot{x}_{2}=x_{3}+a+b_{0} u_{t}, \\
& \dot{x}_{3}=h,
\end{aligned}
$$

where $\quad x_{1}=\psi, \quad x_{2}=r, \quad x_{3}=(K / T) \delta_{w}, \quad a=-(1 / T) r$, $b_{0}=(K / T)$, and $u_{t}=\delta_{c}-\sigma$.

Assumption 1. There exists a positive constant $h^{*}$ satisfying $\|h\| \leq h^{*}$.

Remark 1. Assumption 1 is reasonable because of the environmental disturbances assumed to be bounded. a represents the plant dynamics, $x_{3}$ represents the environmental disturbances, $h$ represents the differential of environmental disturbances, and $u_{t}$ is input signal.

Expression (15) can be rewritten in terms of the matrix as follows:

$$
\dot{x}=A x+B u+E h,
$$

where $x=\left[x_{1}, x_{2}, x_{3}\right]^{T}$ is the extended state vector, 


$$
\begin{aligned}
& A=\left[\begin{array}{ccc}
0 & 1 & 0 \\
0 & -\frac{1}{T} & 1 \\
0 & 0 & 0
\end{array}\right], \\
& B=\left[\begin{array}{l}
0 \\
b_{0} \\
0
\end{array}\right], \\
& E=\left[\begin{array}{l}
0 \\
0 \\
1
\end{array}\right] .
\end{aligned}
$$

Consider an ESO as follows:

$$
\dot{\hat{x}}=A \widehat{x}+B u+L e,
$$

where $e=x_{1}-\widehat{x}_{1}, \widehat{x}=\left[\widehat{x}_{1}, \widehat{x}_{2}, \widehat{x}_{3}\right]^{T}$ is the estimation of $x$, and $L=\left[k_{1}, k_{2}, k_{3}\right]^{T}$ is observer gain matrix with $k_{i}, i=$ $1,2,3$ is positive constant.

Subtracting equation (18) from equation (16), the following can be attained:

$$
\dot{\tilde{x}}=A \tilde{x}+E h-L e,
$$

where $\tilde{x}=x-\widehat{x}$.

Define that $L e=L C \tilde{x}$ with $C=[1,0,0]$. Hence, equation (19) can be rewritten as follows:

$$
\dot{\tilde{x}}=(A-L C) \tilde{x}+E h .
$$

Select the appropriate matrix $L$ to stabilize the matrix $A-L C$. Under Assumption 1, dynamic (20) shows observer (18) ensures that the estimation of the environmental disturbances converges to its real value in a finite time.

\section{Controller Design}

Auxiliary dynamic system is constructed to handle the input constraint in [21], in here, the auxiliary dynamic system is designed as

$$
\begin{aligned}
& \dot{\beta}_{1}=-L_{1} \beta_{1}+\beta_{2}, \\
& \dot{\beta}_{2}=-L_{2} \beta_{2}-b_{0} \sigma,
\end{aligned}
$$

where $L_{1}$ and $L_{2}$ are positive constants.

The controller design process is presented.

Step 1. Define the surface error vector as

$$
z_{1}=\widehat{\psi}-\psi_{d}-\beta_{1} \text {, }
$$

and choose the Lyapunov candidate function as

$$
V_{1}=\frac{1}{2} z_{1}^{2} \text {. }
$$

Along (23) and (21), the time derivative of $V_{1}$ is represented by

$$
\dot{V}_{1}=z_{1} \dot{z}_{1}=z_{1}\left(\widehat{r}-r_{d}+L_{1} \beta_{1}-\beta_{2}\right),
$$

where $\widehat{r}$ is the estimate of $r$.

Define the second surface error as

$$
z_{2}=\widehat{r}-\bar{\alpha}-\beta_{2}
$$

where $\bar{\alpha}$ is the signals derived from the following first-order low-pass filters $\iota_{2} \dot{\bar{\alpha}}+\bar{\alpha}=\alpha$ with $\iota_{2}$ is a positive constant and $\alpha$ is a virtual control defined as

$$
\alpha=-c_{1} z_{1}+r_{d}-L_{1} \beta_{1},
$$

where $c_{1}>0$ is a constant.

Synthesizing with equations (25) and (27),

$$
\dot{V}_{1}=-c_{1} z_{1}^{2}+z_{1}\left(z_{2}+\widetilde{\alpha}\right)
$$

where $\widetilde{\alpha}=\bar{\alpha}-\alpha$.

Step 2. Choose a Lyapunov function as follows:

$$
V_{2}=V_{1}+\frac{1}{2} z_{2}^{2}
$$

From (18), (22), and (26), the following can be attained:

$$
\dot{z}_{2}=-\frac{1}{T} \widehat{x}_{2}+\widehat{x}_{3}+b_{0}\left(\delta_{c}-\sigma\right)-k_{2} e-\dot{\bar{\alpha}}+L_{2} \beta_{2}+b_{0} \sigma .
$$

Substituting (30) into the time derivative of $V_{2}$ and synthesizing with equation (28),

$\dot{V}_{2}=-c_{1} z_{1}^{2}+z_{1}\left(z_{2}+\widetilde{\alpha}\right)+z_{2}\left(-\frac{1}{T} \widehat{x}_{2}+\widehat{x}_{3}+b_{0} \delta_{c}-k_{2} e-\dot{\bar{\alpha}}+L_{2} \beta_{2}\right)$.

To stabilize $z_{2}$, a controller is designed as

$$
\delta_{c}=\frac{1}{b_{0}}\left(-z_{1}+\frac{1}{T} \widehat{x}_{2}-\widehat{x}_{3}+\dot{\bar{\alpha}}-c_{2} z_{2}-L_{2} \beta_{2}\right),
$$

where $c_{2}$ is a positive constant.

Substituting (32) into (31), then

$$
\dot{V}_{2}=-c_{1} z_{1}^{2}-c_{2} z_{2}^{2}+z_{1} \tilde{\alpha}-k_{2} z_{2} e .
$$

As shown in [22], it can prove that $\widetilde{\alpha}$ is bounded. Let $\widetilde{\alpha}_{u}$ be the upper bound of $\widetilde{\alpha}$. Using Young's inequality,

$$
\begin{aligned}
z_{1} \widetilde{\alpha} & \leq z_{1} \widetilde{\alpha}_{u} \\
& \leq \frac{1}{2} z_{1}^{2}+\frac{1}{2} \widetilde{\alpha}_{u}^{2}, \\
z_{2} e & \leq \frac{1}{2} z_{2}^{2}+\frac{1}{2} e^{2} \\
& \leq \frac{1}{2} z_{2}^{2}+\frac{1}{2} \widetilde{x}^{T} \tilde{x} .
\end{aligned}
$$


Step 3. Consider the total Lyapunov function candidate as

$$
V=V_{2}+\frac{1}{2} \beta_{1}^{2}+\frac{1}{2} \beta_{2}^{2}+\frac{1}{2} \widetilde{x}^{T} \tilde{x} .
$$

Differentiating $V$ along (20)-(22), (34), and (35), the following can be attained:

$$
\begin{array}{r}
\dot{V} \leq-\left(c_{1}-\frac{1}{2}\right) z_{1}^{2}-\left(c_{2}-\frac{k_{2}}{2}\right) z_{2}^{2}-\left(c_{3}-\frac{k_{2}}{2}\right) \tilde{x}^{T} \tilde{x} \\
-L_{1} \beta_{1}^{2}-L_{2} \beta_{2}^{2}+\beta_{1} \beta_{2}-b_{0} \beta_{2} \sigma+h^{*}+\frac{1}{2} \widetilde{\alpha}_{u}^{2},
\end{array}
$$

where $c_{3}=\lambda_{\min }(A-L C)$ and $h^{*}$ is given according to $\|E h\| \leq h^{*}$.

Define $\bar{\sigma}$ is upper bound of $\sigma$, and using Young's inequality,

$$
\begin{aligned}
\beta_{2} \sigma & \leq \beta_{2} \bar{\sigma} \\
& \leq \frac{1}{2} \beta_{2}^{2}+\frac{1}{2} \bar{\sigma}^{2}, \\
\beta_{1} \beta_{2} & \leq \frac{1}{2} \beta_{1}^{2}+\frac{1}{2} \beta_{2}^{2} .
\end{aligned}
$$

Then, (37) can be rewritten as

$$
\begin{aligned}
\dot{V} \leq & -\left(c_{1}-\frac{1}{2}\right) z_{1}^{2}-\left(c_{2}-\frac{k_{2}}{2}\right) z_{2}^{2}-\left(c_{3}-\frac{k_{2}}{2}\right) \tilde{x}^{T} \tilde{x} \\
& -\left(L_{1}-\frac{1}{2}\right) \beta_{1}^{2}-\left(L_{2}-\frac{1}{2}-\frac{b_{0}}{2}\right) \beta_{2}^{2}+\mu_{1},
\end{aligned}
$$

where $\mu_{1}=h^{*}+(1 / 2) \widetilde{\alpha}_{u}^{2}+\left(b_{0} / 2\right) \bar{\sigma}^{2}$.

Step 4. In the common continuous control schemes, the design has already been complete in Step 3. Here, the ideology of event-trigger control is employed and the triggering condition will be established in this step. During the flow period between two successive triggering instants, zero-order hold is employed to keep $\delta_{c}$ unchanged. The key to a successful ETC design is to select an appropriate trigger condition. The current triggering instant is denoted as $t_{g_{i}}$ and the next triggering instant $t_{g_{i+1}}$ is determined by satisfying the following triggering condition:

$$
t_{g_{i+1}}=\inf \left\{t \in \mathbb{R} \mid t>t_{g_{i}} \wedge W_{a} \geq \gamma V\right\},
$$

where $W_{a}=\left(c_{1}-(1 / 2)\right) z_{1}^{2}+\left(c_{2}-\left(k_{2} / 2\right)\right) z_{2}^{2}$ and $0<\gamma<$ $\lambda_{\min }\left\{\left(c_{1}-(1 / 2)\right),\left(c_{2}-\left(k_{2} / 2\right)\right)\right\}$ is the adjustable variable. Once the triggering condition is satisfied, thus $W_{a} \leq \gamma V$, and it renders

$$
0>-W_{a}>-\gamma V,
$$

and it implies $-W_{a}$ is bounded. Then,

$$
\begin{aligned}
\dot{V} & \leq-W_{a}-\left(c_{3}-\frac{k_{2}}{2}\right) \tilde{x}^{T} \tilde{x}-\left(L_{1}-\frac{1}{2}\right) \beta_{1}^{2}-\left(L_{2}-\frac{1}{2}-\frac{b_{0}}{2}\right) \beta_{2}^{2}+\mu_{1} \\
& \leq-\left(c_{3}-\frac{k_{2}}{2}\right) \tilde{x}^{T} \tilde{x}-\left(L_{1}-\frac{1}{2}\right) \beta_{1}^{2}-\left(L_{2}-\frac{1}{2}-\frac{b_{0}}{2}\right) \beta_{2}^{2}+\mu_{1},
\end{aligned}
$$

from the definition of $V$, it is sure that $V, \widetilde{x}, \beta_{1}$, and $\beta_{2}$ are bounded and convergent. From $W_{a} \leq \gamma V$, then $z_{1}$ and $z_{2}$ are bounded. Owing to the convergence of $V$ proved, then the convergences of $z_{1}$ and $z_{2}$ are proved. Therefore, all signals in the closed-loop are bounded.

Thus, (40) can be rewritten as

$$
\dot{V} \leq-c V+\mu_{1},
$$

where $c$ is a positive constant, and if the triggering condition is satisfied, $c=\lambda_{\min }\left\{\left(c_{3}-\left(k_{2} / 2\right)\right),\left(L_{1}-(1 / 2)\right),\left(L_{2}-\right.\right.$ $\left.\left.(1 / 2)-\left(b_{0} / 2\right)\right)\right\}, \quad$ otherwise $\quad c=\lambda_{\min }\left\{\left(c_{1}-(1 / 2)\right)\right.$, $\left(c_{2}-\left(k_{2} / 2\right)\right),\left(c_{3}-\left(k_{2} / 2\right)\right) \quad\left(L_{1}-(1 / 2)\right),\left(L_{2}-(1 / 2)-\right.$ $\left.\left.\left(b_{0} / 2\right)\right)\right\}$.

The following can be attained:

$$
V(t) \leq\left(V(0)-\frac{\mu_{1}}{c-\gamma}\right) e^{-(c-\gamma) t}+\frac{\mu_{1}}{c-\gamma} .
$$

From the definition of $V$, it can conclude that $z_{1}, z_{2}, \tilde{x}$, $\beta_{1}$, and $\beta_{2}$ are bounded. From (44), $z_{1}$ exponentially converges to the compact set $\Pi=\left\{z_{1}|| z_{1} \mid \leq 2\left(\mu_{1} /(c-\gamma)\right)\right\}$ that can be made arbitrarily small by adjusting $c-\gamma$. Then, it holds $\lim _{t \rightarrow+\infty}\left|\psi-\psi_{d}\right| \leq \varepsilon$, and the control object is completed.

\section{Simulations and Results}

In this section, comparison with normal PID controller (PIDC) is given through Matlab simulation, and simulation result is provided to validate the effectiveness and feasibility of the proposed controller. The parameters for ESO are set to $k_{1}=4, k_{2}=5$, and $k_{3}=10$. The rudder angle is constrained as $|\delta| \leq 35^{\circ}$. The sampling period of trigger condition is $0.01 \mathrm{~s}$, i.e., $t_{g_{i+1}}-t_{g_{i}}=0.01 . \gamma=0.01$, the reference heading signal is set as $\psi_{d}=30^{\circ}$ for $0 \mathrm{~s} \leq t \leq 150 \mathrm{~s}, \psi_{d}=0^{\circ}$ for $150 \mathrm{~s} \leq t \leq 300 \mathrm{~s}$.

The output responses of the proposed controller are plotted in Figure 2, where it shows that the actual yaw of the surface ship tracks the reference yaw accurately. By comparison with normal PIDC, ETC has the problems of tiny jitter. Figure 3 shows that ETC has a shock in the yaw rate, and the yaw rate is a smooth curve varying with time under PIDC, and the estimation of the yaw rate converges nicely to the actual yaw rate. Figure 4 describes the environmental disturbances and estimation, and it shows that the ESO can accurately estimate the environmental disturbances. Figure 5 shows the rudder angle $\delta$ varying with time under ETC and PIDC, and the local enlarged diagrams of control input $\delta$ around $65 \mathrm{~s}$ for the rudder is shown. The rudder angle $\delta$ is updated 30,000 times under PIDC and 8,512 times under ETC. Though the simulation on the system control part carries on the contrast analysis, PID control strategy reflects the good control performance, but it is hard to implement. 


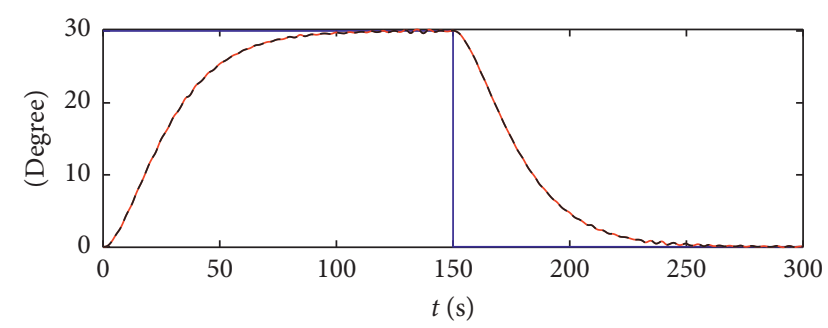

$-\psi_{\mathrm{d}}$
$-\psi$ under ETC
$--\bar{\psi}$

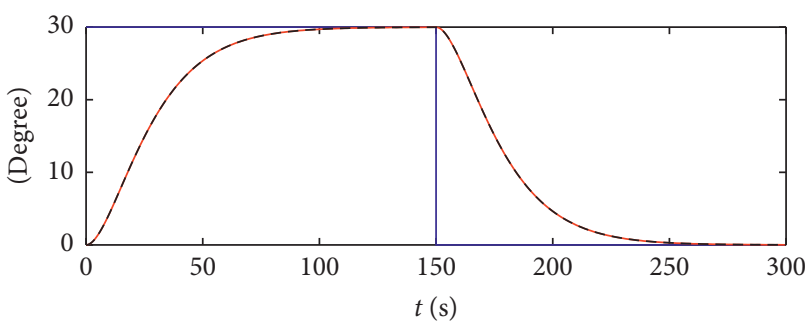

$-\psi_{\mathrm{d}}$
$-\quad \psi$ under PIDC
$---\hat{\psi}$

FIGURE 2: Simulation of heading angle tracking under ETC and PIDC.
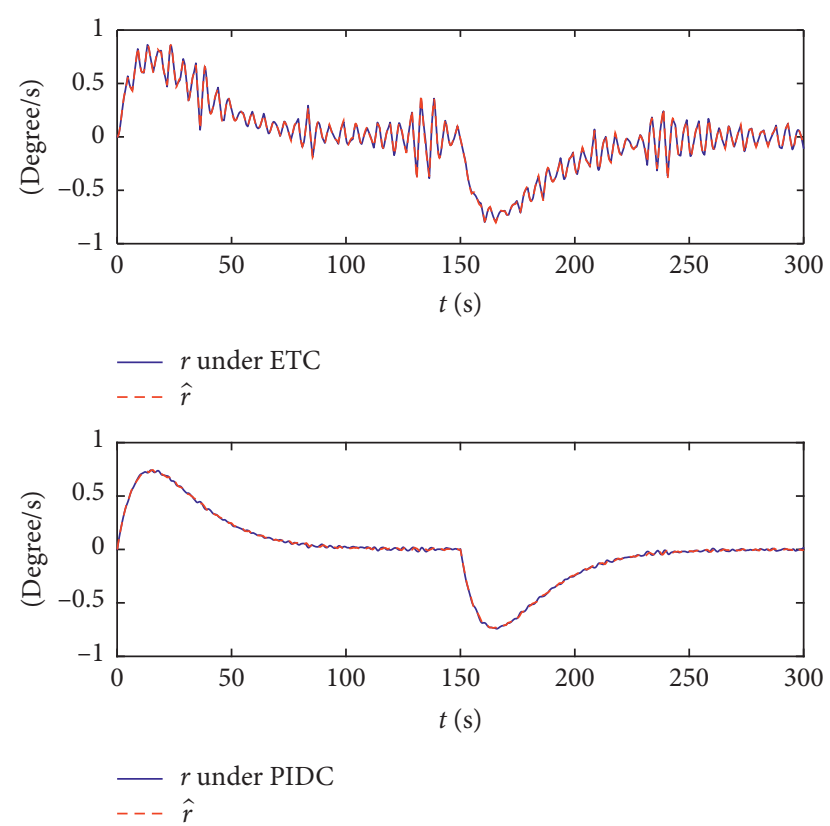

FIgUre 3: Yaw rate $r$ under ETC and PIDC and estimation $\widehat{r}$.

The ETC is easier to implement and energy is saved by the reduction in the rudder rate. In Figure 3, the yaw rate suffers from sudden jumps. To solve this problem, the actuator magnitude and rate limits can be considered as a part of future works.

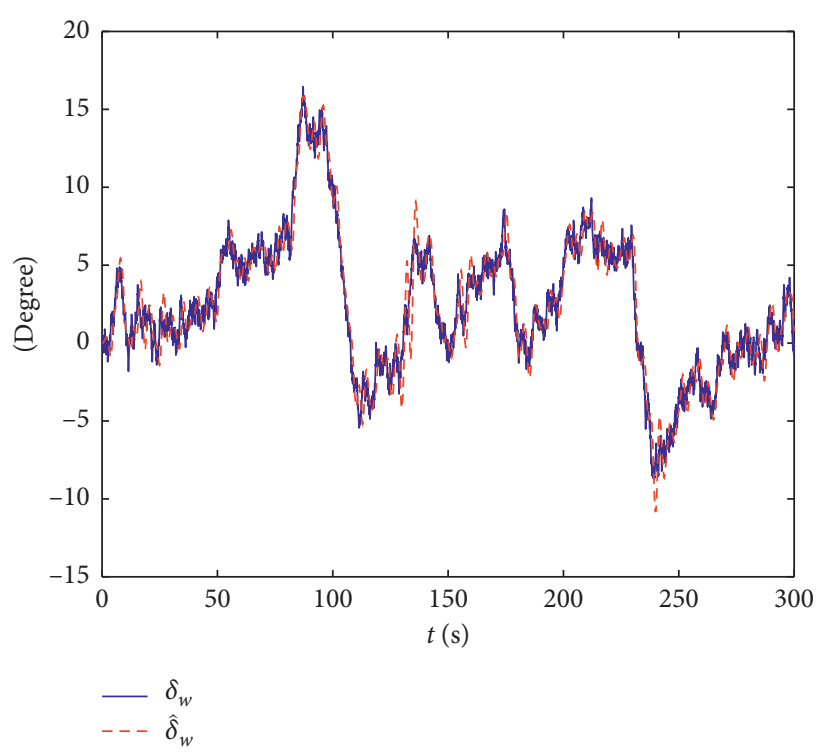

Figure 4: Actual disturbances (blue solid line) and estimation (red dotted line).
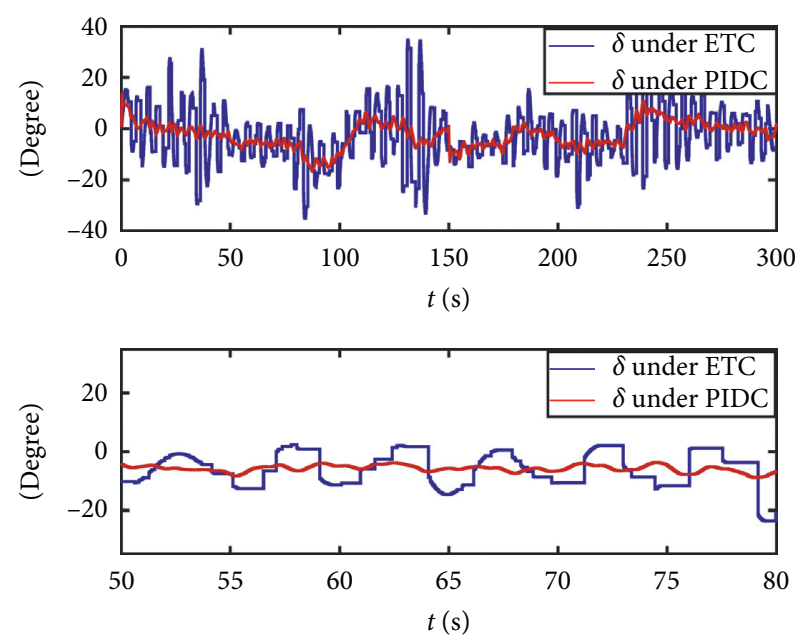

Figure 5: Rudder action under ETC (blue line) and PIDC (red line) and local enlarged diagram around $65 \mathrm{~s}$.

\section{Conclusions}

This paper addresses the autopilot design for a surface vehicle in the presence of environmental disturbances and input saturation. The control architecture is proposed based on ESO and the event-triggered strategy. The ESO is employed to reconstruct the yaw rate. Simulation result shows the designed controller can ensure that the actual yaw of the surface ship tracks the reference yaw accurately. A stability analysis based on Lyapunov direct method proves that all signals are bounded and tracking errors converge to a neighborhood of the origin. 


\section{Data Availability}

The data used to support the findings of this study are included within the article.

\section{Conflicts of Interest}

The authors declare that they have no conflicts of interest.

\section{Acknowledgments}

This study was supported by the 7th Generation Ultra DeepWater Drilling Unit Innovation Project.

\section{References}

[1] J. V. Arnerongen, "Adaptive steering of ships: a model reference approach," Automatica, vol. 20, no. 1, pp. 3-14, 1984.

[2] B. Li, K. L. Teo, K. Lay Teo, C.-C. Lim, and G. Ren Duan, “An optimal PID controller design for nonlinear constrained optimal control problems," Discrete \& Continuous Dynamical Systems-B, vol. 16, no. 4, pp. 1101-1117, 2011.

[3] Y. Zhang and M. Chen, "Application of improved grey predictive control adaptive PID control algorithms in temperature control," Advances in Intelligent Systems and Computing, pp. 382-387, Springer, Berlin, Germany, 2020.

[4] A. Witkowska, M. Tomera, and R. Śmierzchalski, "A backstepping approach to ship course control," International Journal of Applied Mathematics and Computer Science, vol. 17, no. 1, pp. 73-85, 2007.

[5] Y. Wang, S. Chai, and H. D. Nguyen, "Unscented Kalman filter trained neural network control design for ship autopilot with experimental and numerical approaches," Applied Ocean Research, vol. 85, pp. 162-172, 2019.

[6] S. Das, "Applicability of sliding mode control in autopilot design for ship motion control," in Proceedings of the 2014 IEEE International Conference on Recent Advances and Innovations in Engineering, pp. 1-6, IEEE, Jaipur, India, May 2014.

[7] Z. Peng, Y. Tian, and D. Wang, "Autopilot design for a robotic unmanned surface vehicle," in Proceedings of the 34th Chinese Control Conference (CCC), Hangzhou, China, 2015.

[8] G. Rigatos and S. Tzafestas, "Adaptive fuzzy control for the ship steering problem," Mechatronics, vol. 16, no. 8, pp. 479-489, 2006.

[9] L. Liu, D. Wang, and Z. Peng, "Output feedback adaptive control for autopilot design of an unmanned surface vehicle," in Proceedings of the 2015 International Conference on Information Science \& Technology, IEEE, Changsha, China, April 2015.

[10] M. Ejaz and N. Chen, "Sliding mode control design of a ship steering autopilot with input saturation," International Journal of Advanced Robotics Systems, vol. 14, no. 3, 2017.

[11] G. Xia, X. Shao, A. Zhao, and H. Wu, "Adaptive neural network control with backstepping for surface ships with input dead-zone," Mathematical Problems in Engineering, vol. 2013, Article ID 530162, 9 pages, 2013.

[12] K. D. Do, "Practical control of underactuated ships," Ocean Engineering, vol. 37, no. 13, pp. 1111-1119, 2010.

[13] Y. Zhao and L. Dong, "Robust path-following control of a container ship based on serret-frenet frame transformation," Journal of Marine Science and Technology, vol. 25, no. 1, pp. 69-80, 2019.
[14] C.-Y. Tzeng and K.-F. Lin, "Adaptive ship steering autopilot design with saturating and slew rate limiting actuator," International Journal of Adaptive Control and Signal Processing, vol. 14, no. 4, pp. 411-426, 2000.

[15] A. R. Babaei, M. Mortazavi, and M. H. Moradi, "Classical and fuzzy-genetic autopilot design for unmanned aerial vehicles," Applied Soft Computing, vol. 11, no. 1, pp. 365-372, 2011.

[16] H. Katayama and H. Aoki, "Straight-line trajectory tracking control for sampled-data underactuated ships," IEEE Transactions on Control Systems Technology, vol. 22, no. 4, pp. 1638-1645, 2014.

[17] H. Katayama and H. Aoki, "Experimental evaluation of supervisory switching control for unmanned surface vehicles," IEEE Journal of Oceanic Engineering, vol. 44, no. 1, pp. 7-28, 2019.

[18] N. Bialystocki and D. Konovessis, "On the estimation of ship's fuel consumption and speed curve: a statistical approach," Journal of Ocean Engineering and Science, vol. 1, no. 3, pp. 157-166, 2016.

[19] Y. Deng, X. Zhang, I. Namkyun, G. Zhang, and Q. Zhang, "Event-triggered robust fuzzy path following control for underactuated ships with input saturation," Ocean Engineering, vol. 186, no. 15, 2019.

[20] T. I. Fossen, Marine Control Systems, Marine Cybernetics, Trondheim, Norway, 2002.

[21] G. Xia, C. Sun, B. Zhao, X. Xia, and X. Sun, "Neuroadaptive distributed output feedback tracking control for multiple marine surface vessels with input and output constraints," IEEE Access, vol. 7, pp. 123076-123085, 2019.

[22] G. Xia, C. Sun, B. Zhao, and J. Xue, "Cooperative control of multiple dynamic positioning vessels with input saturation based on finite-time disturbance observer," International Journal of Control, Automation and Systems, vol. 17, no. 2, pp. 370-379, 2019. 